\title{
eJRIEPS
}

Ejournal de la recherche sur l'intervention en éducation physique et sport

$26 \mid 2012$

Varia

\section{Pour une didactique clinique de l'EPS : Perspectives pour la formation des enseignants}

\section{Marie-France Carnus}

\section{(Q) OpenEdition \\ Journals}

Édition électronique

URL : http://journals.openedition.org/ejrieps/3342

DOI : 10.4000/ejrieps.3342

ISSN : 2105-0821

Éditeur

ELLIADD

\section{Référence électronique}

Marie-France Carnus, « Pour une didactique clinique de l'EPS : Perspectives pour la formation des enseignants », eJRIEPS [En ligne], 26 | 2012, mis en ligne le 01 avril 2012, consulté le 15 janvier 2020. URL : http://journals.openedition.org/ejrieps/3342 ; DOI : 10.4000/ejrieps.3342

\section{(0) (1)}

La revue eJRIEPS est mise à disposition selon les termes de la Creative Commons Attribution 4.0 International License. 


\section{Pour une didactique clinique de I'EPS}

\section{Perspectives pour la formation des enseignants}

\section{Marie-France Carnus}

IUFM Midi-Pyrénées, Ecole interne de l'Université de Toulouse 2-Le Mirail, UMR EFTS, Université de Toulouse

\section{Résumé}

Cet article présente en trois parties l'orientation scientifique dans laquelle j'inscris aujourd'hui mon activité de recherche: la didactique clinique de l'EPS. D'abord, je rappelle, à travers mon parcours singulier, l'émergence progressive de ce positionnement scientifique, puis, je me focalise sur mes principaux points d'appui et constructions théoriques et méthodologiques progressivement élaborées, enfin, j'évoque les principales orientations de recherche qui soutiennent aujourd'hui mon activité de chercheure. Cette trajectoire et les orientations qui en découlent ne peuvent se comprendre qu'à travers un cheminement singulier qui caractérise mon épistémologie de chercheure. Au cœur des sujets et centrées sur le fonctionnement didactique, ces orientations rejoignent des préoccupations actuelles relatives à la fécondité des articulations entre recherche et formation des enseignants et se proposent d'investiguer la dimension heuristique de la notion de compétence. Inscrites dans une double filiation, ces orientations, qui rencontrent aujourd'hui l'intérêt d'étudiants, soutiennent mon désir de chercheure afin de poursuivre ma route, avec d'autres.

\section{Introduction}

L'orientation scientifique dans laquelle j'inscris aujourd'hui mon activité de recherche est la didactique clinique de l'éducation physique et sportive (EPS). Cette orientation se situe dans la croisée de la didactique de l'EPS et d'une clinique du sujet.

D'une part, la didactique de l'EPS correspond à mon ancrage d'origine. C'est un domaine en évolution depuis les années 70-80 dans lequel se développent des recherches autour de problématiques élaborées au regard de la spécificité de contenus disciplinaires résultant de la transposition, dans le champ scolaire, de pratiques sociales de référence : les activités physiques sportives et artistiques (APSA). Ces contenus, essentiellement mais non exclusivement moteurs enrichissent l'expérience corporelle de chaque élève et 


\section{eJRIEPS 26 avril 2012}

contribuent à la formation d'un citoyen lucide, cultivé, autonome et responsable. D'autre part, une clinique du sujet d'orientation psychanalytique qui prend à son compte l'hypothèse freudienne selon laquelle « le moi n'est pas maître dans sa propre maison » (Freud, 1981). Ce positionnement, que j'ai progressivement construit au cours de ces dix dernières années, conduit à considérer le savoir comme un élément à la fois endogène et exogène dans la relation didactique. Dans le prolongement de ma Note de synthèse (Carnus, 2009a), je souhaiterais ici montrer que cette orientation est de nature à renouveler les problématiques de recherches dans le champ de la didactique - plus particulièrement en EPS - et plus largement en Sciences de l'Education. Je voudrais aussi montrer que cette orientation est de nature à ouvrir des perspectives fécondes, heuristiques et originales pour la formation des enseignants à condition de se situer dans une vision non applicationniste, distanciée et critique.

Cette présentation comprendra trois parties. D'abord, je rappellerai, à travers mon parcours singulier, l'émergence progressive de ce positionnement scientifique, puis, je me focaliserai sur mes principaux points d'appui et constructions théoriques et méthodologiques progressivement élaborées, enfin, je présenterai les principales orientations de recherche qui soutiennent aujourd'hui mon activité de chercheure.

\section{L'émergence progressive de mon positionnement scientifique}

Mon positionnement scientifique actuel n'est pas la résultante d'une évolution linéaire. A y regarder de près, cette évolution s'origine bien avant la thèse que j'ai soutenue en mars 2001 (Carnus, 2001). Je me limiterai ici à considérer la période comprise entre 1978 et aujourd'hui (figure 1). 


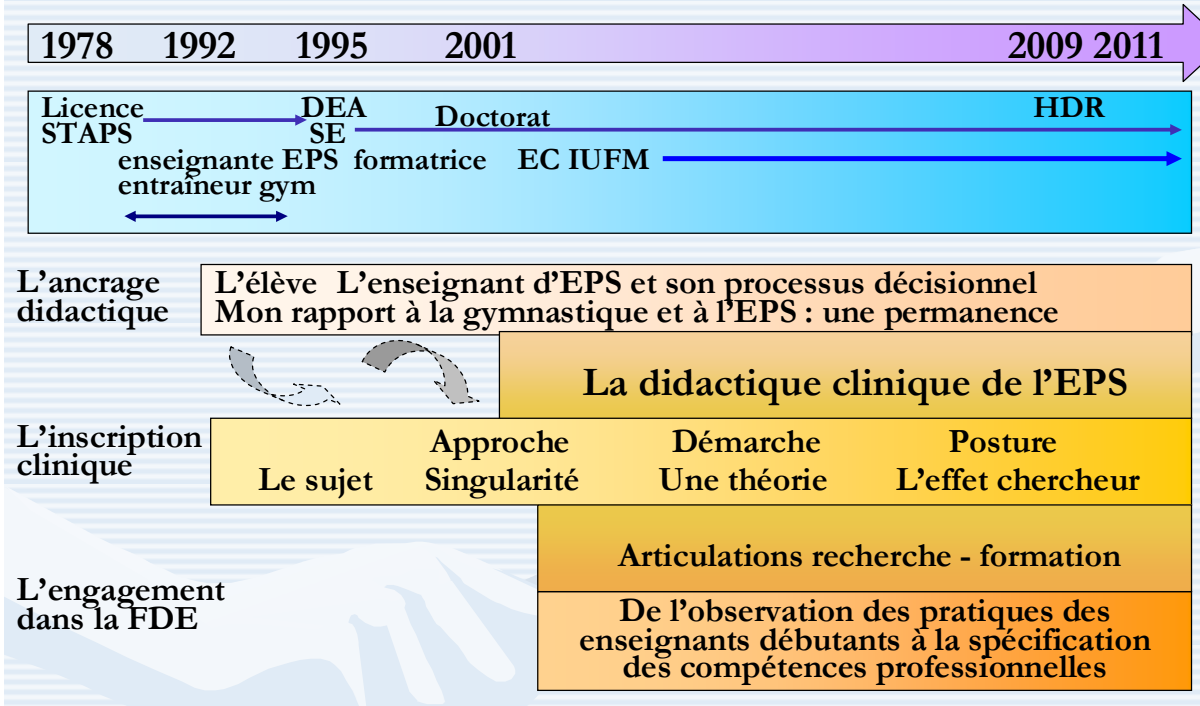

\section{Une dynamique singulière qui s'ancre dans un déjà-là}

Figure 1. L'émergence progressive de mon positionnement scientifique.

1978, date à laquelle j'ai obtenu la licence STAPS, ce qui a orienté dès le départ ma trajectoire scientifique. Dans la conjoncture d'une reprise d'études tardive, 14 ans plus tard, au fil des rencontres scientifiques, j'ai opéré ce que j'ai appelé une «bascule » en Sciences de l'Education en 1995. Mon questionnement de recherche s'est aussi en parallèle nourrit de mon expérience professionnelle d'enseignement de l'EPS, de formation des enseignants d'EPS, d'entraîneure en gymnastique avant que j'intègre à part entière l'IUFM en 2002 en tant qu'enseignant chercheur. Ainsi, au fil des institutions traversées, les racines de cet espace temporel sont enfouies dans une histoire personnelle antérieure. Toutefois, mon positionnement scientifique et les prolongements que j'entrevois aujourd'hui ne peuvent se comprendre qu'au travers de l'imbrication des trois volets qui forment l'ossature de ma Note de synthèse: l'ancrage didactique, l'inscription clinique et l'engagement dans la formation des enseignants.

En lien étroit avec mon rapport personnel à la gymnastique et à l'EPS, l'ancrage didactique trace une ligne de force permanente dans ma recherche. D'abord centrés sur l'activité perceptivo-motrice des élèves confrontés aux apprentissages gymniques, mes travaux se sont focalisés sur le processus décisionnel de l'enseignant d'EPS en gymnastique au travers de l'analyse des écarts entre intention et décision, écarts révélateurs de remaniements des contenus d'enseignement. Mes travaux de doctorat ont notamment montré qu'une part de ces remaniements s'opère à l'insu des enseignants. 


\section{eJRIEPS 26 avril 2012}

Centrée sur le suivi des enjeux de savoir dans le cadre d'une relation ternaire, la recherche en didactique de l'EPS ne pouvait, me semble-t-il, exclure ce phénomène inconscient de ses réflexions. Pour André Terrisse ce travail est le véritable déclencheur de l'option clinique en didactique de l'EPS (Terrisse, 2009).

Toutefois, au fil des relectures dans l'après-coup de mes premières publications, je me suis aperçue que cette sensibilité clinique m'habite dès le départ. J'ai identifié trois graduations dans l'inscription dans cette dimension: l'approche clinique qui intègre la singularité du sujet ; la démarche clinique conduisant à l'élaboration d'une théorie du sujet didactique ; la posture clinique marquée par la reconnaissance d'un effet-chercheur pour, dans et par la recherche.

Le dernier volet dans lequel je suis fortement impliquée à différents niveaux depuis 2002 est relatif à la formation des enseignants. Tout en développant des problématiques propres aux aspects disciplinaires, dans une approche comparatiste en didactique, le cheminement au sein d'une équipe de recherche technologique en IUFM ${ }^{1} \mathrm{~m}^{\prime}$ a conduite à extraire de l'observation des pratiques d'enseignants débutants des indices relatifs à la spécification des compétences professionnelles et à approfondir une réflexion de nature épistémologique sur les liens envisageables entre recherche et formation.

Ainsi, l'émergence progressive de mon positionnement scientifique résulte d'une dynamique singulière qui s'ancre dans un déjà-là. Cette dynamique a été soutenue au sein de trois équipes (l'AP3E ${ }^{2}$ aujourd'hui $\mathrm{EDiC}^{3}$; le GRIDIFE$^{4}$ et le groupe codisciplinaire ${ }^{5}$ ), équipes dans lesquelles les aventures humaines et scientifiques que j'ai vécues et que je vis encore ont renforcé ma croyance en la puissance productrice que peut engendrer un collectif de chercheurs.

\section{Mes points d'appui et mes élaborations théoriques et méthodologiques}

3. 1. Epistémologie de la didactique clinique de l'EPS

L'émergence de la didactique clinique de l'EPS repose sur le constat de l'insuffisance des cadres didactiques actuels pour lire certains phénomènes relatifs à la transmission appropriation de savoirs en EPS (Terrisse, Carnus, 2009).

\footnotetext{
${ }^{1}$ GRIDIFE, ERTé 46 puis ERT 64.

${ }^{2}$ Analyse des Pratiques Enseignantes et de leurs Effets sur l'apprentissage des Elèves.

${ }^{3}$ Equipe de Didactique Clinique de l'EPS.

${ }^{4}$ Groupe de Recherche sur les Interactions Didactique et la Formation des Enseignants

${ }^{5}$ Groupe animé par Claudine Blanchard-Laville et constitué de : Claudine Blanchard-Laville, LouisMarie Bossard, Sylvain Broccolichi, Marie-France Carnus, Philippe Chaussecourte, Eric Roditti, Catherine
} Verdier. 


\section{eJRIEPS 26 avril 2012}

J'illustre mes propos. Au cours de l'observation filmée d'une leçon de gymnastique en collège, je constate qu'une enseignante expérimentée d'EPS, spécialiste en gymnastique, modifie soudainement l'agencement du milieu didactique en déplaçant un tapis. Au cours de l'entretien d'après-coup, cette décision est justifiée par l'enseignante par ce qui apparaît comme un « impossible à supporter » constitutif de l'épistémologie personnelle et professionnelle de cette enseignante qui redoute en permanence l'accident. Le croisement des différentes analyses met en évidence que, si cette décision a pour conséquence de compromettre la construction des savoirs initialement visés, elle semble néanmoins permettre à l'enseignante de gérer, sur le moment, l'angoisse provoquée par la lecture des comportements de certains élèves dans une situation qu'elle a elle-même mise en place conformément à son intention. Ce phénomène semble relever de mécanismes inconscients à l'œuvre dans le fonctionnement didactique et illustre de manière empirique le positionnement de la didactique clinique de l'EPS dans une double filiation : celle de la didactique de l'EPS et celle de la clinique d'orientation psychanalytique en Sciences de l'Education.

Ce positionnement théorique et épistémologique questionne la nature des relations que la didactique clinique de l'EPS peut entretenir avec les didactiques des autres disciplines, notamment celle des mathématiques, particulièrement féconde, ainsi qu'avec la didactique comparée en émergence depuis ces dix dernières années. L'ancrage didactique du champ conduit en effet à des emprunts, des importations de concepts et de méthodes qui méritent nécessairement d'être revisités au regard de la spécificité disciplinaire de l'EPS ainsi qu'au regard de l'orientation clinique : une clinique du sujet qui se différencie d'une clinique des situations ou d'une clinique de l'activité. Ce travail nomologique relatif au positionnement de ce champ en émergence m'apparaît nécessaire pour en assoir davantage la scientificité et représente un des axes forts de mon projet.

En même temps il convient de se questionner sur la nature des articulations entre recherche et formation afin de dégager des perspectives originales pour la formation des enseignants (figure 2). 


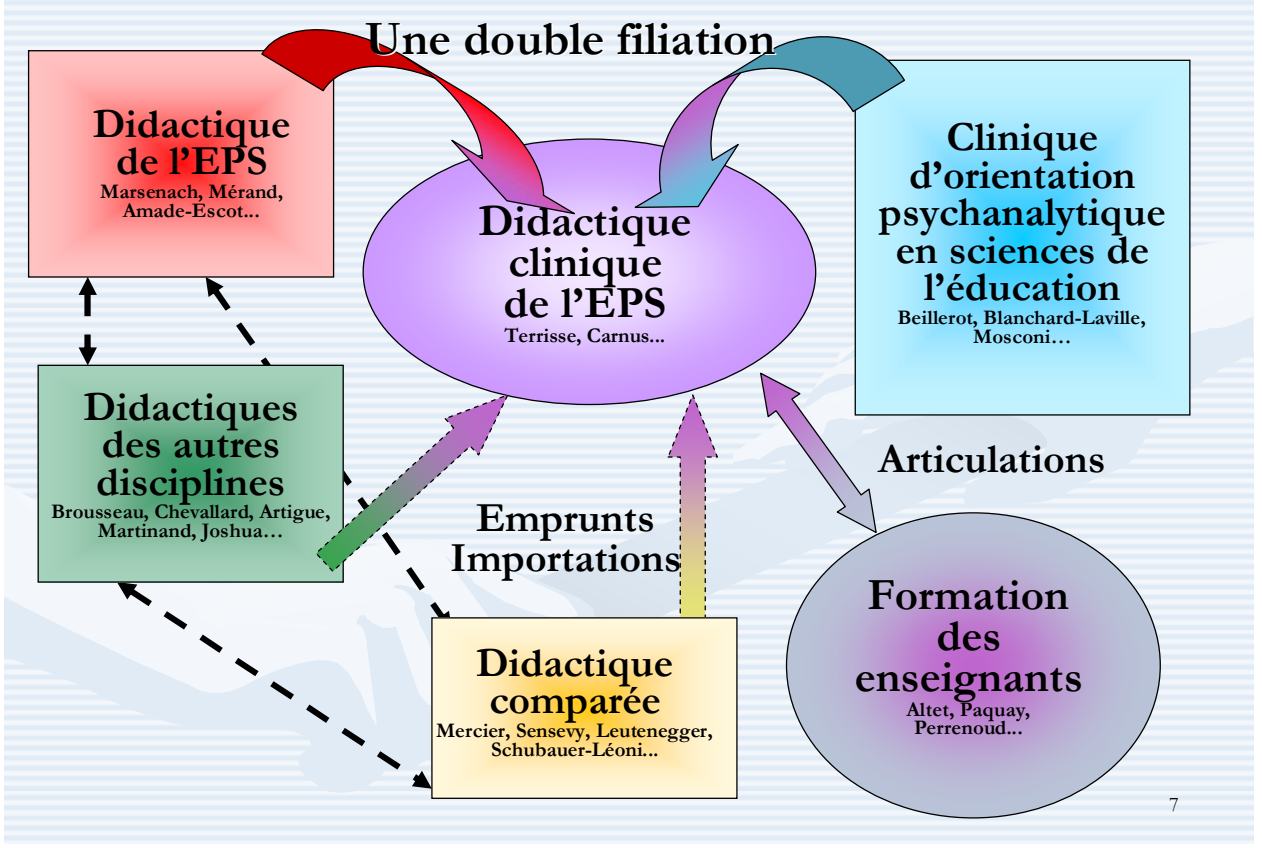

Figure 2: Le positionnement théorique et épistémologique de la didactique clinique de l'EPS.

3. 2. Les articulations entre recherche et formation

Récemment, j'ai eu l'occasion d'approfondir cette question (Carnus, 2009b). Ces deux champs sociaux diffèrent notamment par leurs enjeux, leurs règles de fonctionnement, leurs temporalités ainsi que leurs acteurs. De manière schématique, ces articulations peuvent se situer au niveau des concepts tels qu'ils sont revisités (par exemple la transposition didactique ou encore le milieu didactique); des méthodes (par exemple la méthodologie d'ingénierie didactique); et des résultats (par exemple les écarts entre intention et décision). Ainsi, la recherche en didactique clinique de l'EPS peut, sous réserve d'en créer les conditions, nourrir la formation jouant ainsi une fonction sociale et professionnelle à la fois compréhensive, prospective et intégrative.

Pour aller plus loin dans cette dialectique, ces différentes articulations devraient me semble-t-il être questionnées en retour par les acteurs de la formation exerçant alors une fonction critique et productive pour la recherche. Ce va et vient entre recherche et formation est de nature à renouveler les problématiques de recherche et les dispositifs de formation.

A partir de ces points d'appui, je voudrais citer quelques unes de mes élaborations qui ont contribué au sein de l'EDiC à l'émergence de ce champ me permettant d'y creuser mon sillon.

3. 3. Mes principales élaborations théoriques et méthodologiques 


\section{eJRIEPS 26 avril 2012}

3. 3. 1. Le processus décisionnel de l'enseignant

Dans l'après-coup de la lecture de ma Note de synthèse, je me suis aperçue que je n'avais pas développé les avancées de mes travaux portant sur le processus décisionnel, objet de recherche au cœur de ma thèse. Cet article me fournit l'occasion de revenir sur cet objet que j'ai particulièrement retravaillé et qui a donné lieu à un chapitre dans l'ouvrage codirigé avec André Terrisse (Terrisse, Carnus, 2009 ; Carnus, 2009c).

La décision de l'enseignant n'est que la partie visible d'un processus. Entre concession et renoncement, la reprise des corpus de ma thèse, m'a permis de différencier trois types de décisions didactiques : la nécessité de trancher, le compromis et la compromission mettant en avant la complexité et la dynamique singulière de l'activité décisionnelle de l'enseignant d'EPS et ses effets sur les savoirs enseignés (Carnus, 2009c).

3. 3. 2. Concepts et méthodes des didactiques revisités

Une autre élaboration est relative au concept princeps et fondateur de nombreuses didactiques disciplinaires: la transposition didactique qui représente un puissant outil d'analyse du fonctionnement didactique. Son importation m'a amené à le questionner. II en ressort une tentative de schématisation qui permet d'en dépasser une vision linéaire en mettant au centre la question des écarts (figure 3). Cette schématisation que, depuis la rédaction de la Note de synthèse, j'ai remaniée met en tension trois sources d'influences s'exerçant à tout moment sur les enjeux de savoir : sociale, institutionnelle, personnelle. Ces influences et leurs fluctuations dans l'avancée du temps didactique apportent une explication au caractère labile et contingent de la Référence, particulièrement développée dans la thèse de Frédéric Heuser (Heuser, 2009). 


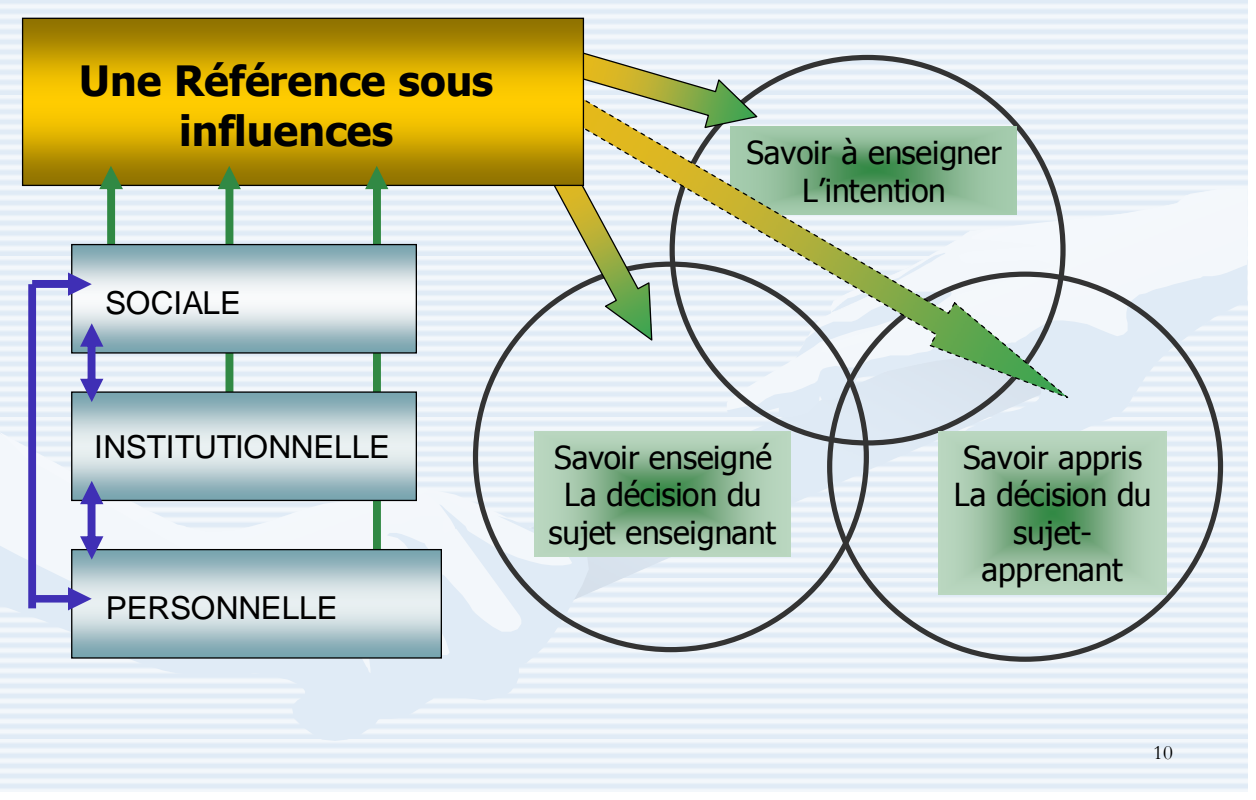

Figure 3. La transposition didactique : un outil d'analyse du fonctionnement didactique.

J'ai également revisité la méthodologie d'ingénierie didactique dont le schéma chronologique en phase avec la temporalité construite en didactique clinique de l'EPS (le déjà-là, l'épreuve et l'après-coup) permet d'accéder aux écarts entre intention et décision. Dans le cadre d'études de cas croisées le rapprochement des données m'apparaît plus fiable dans la mesure où un certain nombre de variables sont en partie contrôlées a priori par le chercheur. En résonnance avec les travaux de Georges Devereux (Devereux, 1980), j'ai aujourd'hui conscience que ce dispositif méthodologique représente un ensemble de manœuvres défensives mises en place par le chercheur pour pallier l'angoisse provoquée par les données qu'il recueille. La posture clinique amène à questionner l'effet chercheur qui renvoie aux perturbations transférentielles et contre transférentielles occasionnées par la mise en place de ce dispositif (Blanchard-Laville, 2007).

De la même façon, j'ai interrogé le milieu didactique et la mésogenèse en tant que concepts nécessaires pour l'étude du fonctionnement didactique permettant de rendre compte des écarts entre intention et décision à chaque étape de la transposition didactique.

3. 3. 3. Une théorie du sujet didactique 


\section{eJRIEPS 26 avril 2012}

Ce cheminement m'a conduite à élaborer une théorie du sujet didactique sous-jacente afin d'articuler les dimensions didactique et clinique de ce champ en émergence. Trois présupposés inter reliés fondent et alimentent cette élaboration théorique. Le sujet didactique est singulier, unique, marqué par son histoire et responsable de ses choix. Le deuxième présupposé est relatif aux assujettissements aux diverses institutions traversées de nature à engendrer un jeu complexe de codéterminations à la fois ressources et contraintes. Le troisième présupposé intègre la dimension de l'inconscient, source de divisions. Entre une sphère privée et une sphère publique, le sujet didactique ne peut maîtriser ce qu'il ne connaît pas et qui fait sans cesse pression sur lui. Cet ensemble de considérations m'amène à esquisser une formalisation dessinant les contours du champ de la didactique clinique de l'EPS (figure 4).

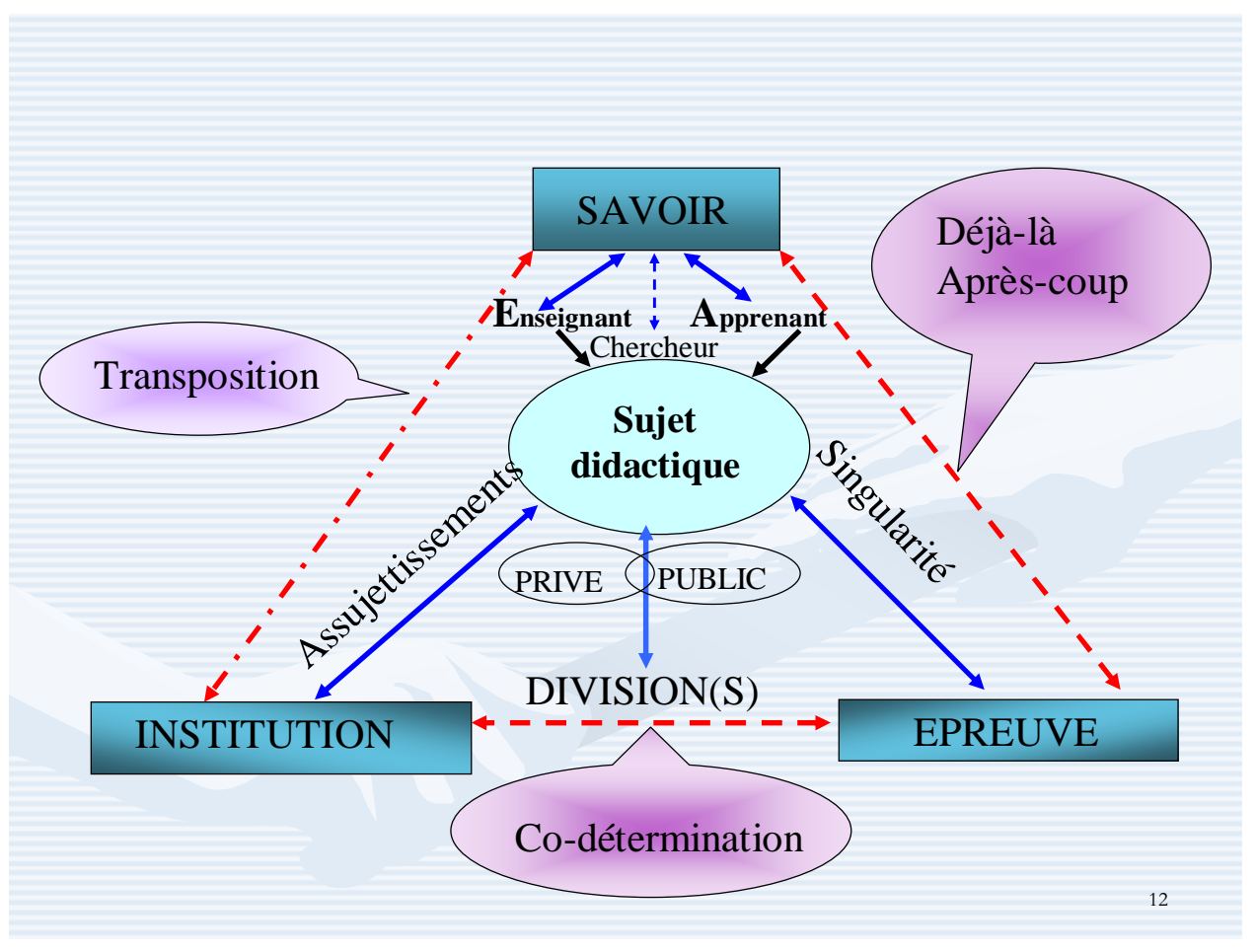

Figure 4. Essai de formalisation de la théorie du sujet didactique.

Au cœur d'une ternarité recomposée par trois instances distinctes et interdépendantes : le savoir, enjeu de la transmission - appropriation ; l'institution dans laquelle le savoir est transposé de manière non linéaire et sous diverses influences ; et l'épreuve, moment de vérité des décisions où il est convoqué, le sujet didactique, enseignant et apprenant entretient un jeu de relations privées, intimes, avec le savoir. Assujetti aux diverses institutions traversées, le sujet didactique dans son irréductible singularité agit dans et par l'épreuve dans laquelle la contingence de l'enseignement et de l'apprentissage ne peut se 


\section{eJRIEPS 26 avril 2012}

comprendre qu'au sein d'une temporalité construite entre un déjà-là conceptuel, intentionnel et expérientiel et un après-coup considéré comme un processus de reconstruction rétroactive dans lequel le sujet remanie les traces mnésiques de ses expériences. Divisé entre une sphère privée et une sphère publique, le sujet didactique prend des décisions dans un espace de liberté qu'il s'autorise ou s'interdit influencé, parfois à son insu, par un jeu de codéterminations qui se révèle inéluctablement dans l'épreuve. La posture clinique amène à intégrer la subjectivité du chercheur et ses effets à différents niveaux. Ceci conduit à questionner de l'intérieur les dimensions éthiques et déontologiques de la recherche. Entre recherche de rationalité et prise en compte de la subjectivité, je fais l'hypothèse que l'objectivité est à chercher dans la reconnaissance de cette tension constitutive de toute recherche.

\section{3. 4. Les problématiques relatives aux enseignants débutants}

En lien avec les problématiques touchant aux enseignants débutants, j'évoquerai la construction d'un outil d'analyse des interactions didactiques langagières dans leurs dimensions verbales et non verbales. Le travail collaboratif au sein du GRIDIFE, particulièrement avec Claudine Garcia-Debanc en didactique du français a permis d'évaluer la pertinence et la généricité d'une double analyse lexicale et sémantique des interactions didactiques. Cet outil permet d'extraire des indicateurs de nature à différencier les pratiques langagières des enseignants débutants et expérimentés.

Enfin, suite à l'ouvrage collectif du GRIDIFE (Carnus, Garcia-Debanc, Terrisse, 2008), j'ai été amenée à construire un « méta cadre » d'analyse des compétences professionnelles des enseignants qui comprend quatre dimensions inter reliées qui interrogent la formation des enseignants et sur lesquelles je vais revenir dans la dernière partie de l'article.

\section{Mes orientations de recherche aujourd'hui}

Ces orientations s'appuient à la fois sur les avancées d'opérations de recherche menées ou en cours ainsi que sur des codirections de masters et de thèses déjà soutenus ou en cours. Elles représentent un ensemble de voies à approfondir dans la perspective de futures directions de recherches.

La figure 4 tente de synthétiser de manière dynamique les orientations du projet scientifique dans lequel je souhaite aujourd'hui m'inscrire. 


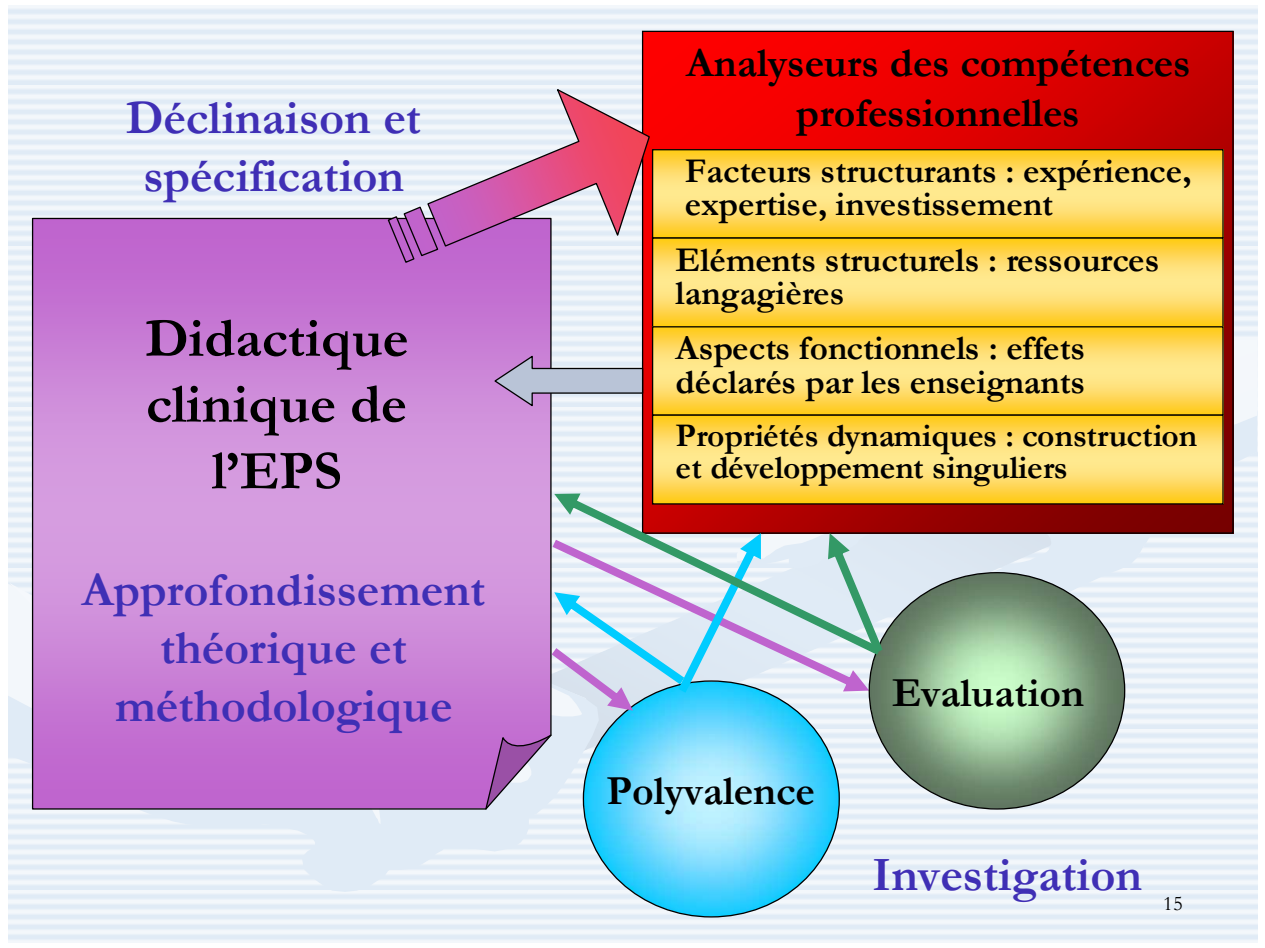

Figure 5. Les orientations de mon projet scientifique.

Un premier axe renvoie à l'approfondissement théorique et méthodologique de la didactique clinique de l'EPS. II vise à poursuivre le travail de formalisation entrepris. II s'inscrit dans le long terme.

A moyen terme, un deuxième axe consiste à décliner et à spécifier les quatre niveaux d'analyse des compétences professionnelles des enseignants. La problématique de l'expérience et de l'expertise est au cœur du travail d'André Touboul (Touboul, 2010). Les études de cas contrastés qu'il mène sont de nature à identifier et à préciser des indicateurs de ces facteurs structurant les compétences professionnelles. En lien avec un investissement dans la transmission du savoir, un troisième facteur qui soutient le désir d'enseigner est à explorer à l'aide d'outils qu'il reste à construire. Concernant les éléments structurels des compétences professionnelles, les prolongements de travaux relatifs aux ressources langagières sont envisagés à plusieurs niveaux: des rapprochements pluridisciplinaires à des fins comparatistes, un approfondissement de l'étude du registre métaphorique en lien avec la signature de l'enseignant (Carnus, GarciaDebanc, 2011). Egalement, la dimension non verbale des interactions langagières est en cours d'exploration grâce à l'utilisation du logiciel Dartfish. Par ailleurs, la question des effets des pratiques enseignantes interroge le niveau fonctionnel des compétences professionnelles. II semblerait qu'au fil du temps, les enseignants expérimentés 


\section{eJRIEPS 26 avril 2012}

développent un sentiment d'insatisfaction et même d'impuissance perceptible à travers les effets déclarés qui diffèrent des effets constatés sur les apprentissages des élèves. Enfin, les propriétés dynamiques à l'œuvre à travers la comparaison entre enseignants débutants et expérimentés, semblent relever de logiques singulières. Néanmoins, le projet est d'extraire au-delà de la singularité des sujets, des régularités fournissant des indices de professionnalisation.

Un dernier axe, à plus court terme est relatif à l'investigation de thématiques particulièrement heuristiques et originales. La première est celle de l'évaluation, dont les dimensions publiques et privées ont marqué l'histoire des sujets, notamment lorsqu'ils étaient élèves. S'appuyant sur un grand nombre de travaux antérieurs touchant à la construction et au développement de la compétence à évaluer chez les enseignants d'EPS, cette investigation se prolonge au sein d'une équipe pluridisciplinaire. Le projet à terme est de déboucher sur la construction d'ingénieries de formation à l'évaluation. Cette thématique est également au cœur du travail de master de Pierre Pédêches qui a approfondi le rôle des procédures et des dispositifs de l'évaluation sur le contrat didactique (Pédèches, 2010). La deuxième thématique est celle de la polyvalence. La réflexion entreprise au sein de l'équipe codisciplinaire animée par Claudine Blanchard-Laville, autour d'un corpus longitudinal relatif aux pratiques enseignantes d'un professeur des écoles ainsi que la direction de la thèse d'Yves Léal portant sur la question de l'éducation à la santé dans un enseignement polyvalent me fournissent aujourd'hui des points d'appui solides pour l'étude de cette compétence au cœur de la problématique de l'enseignant du premier degré.

A l'interface de la recherche et de la formation, l'étude de ces deux thématiques devrait permettre de préciser le cadre d'analyse des compétences professionnelles et renforcer la pertinence d'un regard clinique en didactique de l'EPS.

\section{Conclusion}

J'arrive au terme de cet article. Mon positionnement scientifique actuel et les orientations qui en découlent ne peuvent se comprendre qu'à travers un cheminement singulier qui caractérise mon épistémologie de chercheure. Au cœur des sujets et centrées sur le fonctionnement didactique ces orientations rejoignent des préoccupations actuelles relatives à la fécondité des articulations entre recherche et formation des enseignants et se proposent d'investiguer la dimension heuristique de la notion de compétence. Inscrites dans une double filiation, ces orientations, qui rencontrent aujourd'hui l'intérêt d'étudiants, 


\section{eJRIEPS 26 avril 2012}

peuvent s'appuyer et se développer sur des dynamiques d'équipes dans des institutions renouvelées. Elles soutiennent mon désir de chercheure afin de poursuivre ma route, avec d'autres. Dans un contexte historique de mutation de la formation des enseignants, ce programme de recherche ambitionne, au sein de la communauté des chercheurs en Sciences de l'Education, de soutenir la cause d'une fonction sociale et professionnelle de la recherche dans la formation en même temps qu'une fonction critique et productive de la formation au regard de la recherche.

\section{Bibliographie}

Blanchard-Laville, C. (2007). Introduction au symposium: Regards croisés sur le processus de recherche dans le contexte d'approche clinique d'orientation psychanalytique. Actes du colloque international de l'AREF, 28-31 août 2007, Strasbourg.

Carnus, M.F. (2001). Analyse didactique du processus décisionnel de l'enseignant d'EPS, thèse non publiée, Université Paul Sabatier, Toulouse.

Carnus M.F. (2009a). Pour une didactique clinique de l'EPS. Perspective pour la formation des enseignants. Note de synthèse pour l'habilitation à diriger des recherches. Université Paris Ouest Nanterre la Défense, le 8 décembre 2009

Carnus, M.F. (2009b). Les relations possibles entre recherche sur les pratiques enseignantes et formation professionnelle initiale et continue. Illustrations en didactique de l'éducation physique et sportive (EPS), in J. CLANET (dir), Recherche et formation, quelles articulations ? PUR, Rennes, 57-68.

.Carnus, M.F. (2009c). La décision de l'enseignant en didactique clinique. Etudes de cas en Education Physique et Sportive. In A. Terrisse et MF Carnus (dir). Didactique clinique de I'EPS, Quels enjeux de savoirs ? De Boëck, Bruxelles, 63-81. [

Carnus M.F., Garcia-Debanc C. (2011). Pratiques langagières des enseignants et spécificités des contenus disciplinaires. Analyse comparée en français et en EPS. Actes du deuxième colloque international de l'ARCD, 20-22 janvier 2011, Lille.

Carnus M.F., Garcia-Debanc C., Terrisse A. (2008). Analyse des pratiques des enseignants débutants : approche didactique. La pensée sauvage, Grenoble.

Devereux, G. (1980). De l'angoisse à la méthode dans les sciences du comportement, Flammarion, Paris. 


\section{eJRIEPS 26 avril 2012}

Freud, S. (1981). Une difficulté de la psychanalyse (1917). (Traduction de l'anglais par E. Kestemberg et M. Green). Revue française de psychanalyse, 45, 6.

Heuser, F. (2009). Du savoir enseigné et évalué à la référence de l'enseignant d'EPS : étude didactique clinique en karaté, mémoire de doctorat, Université Paul Sabatier, 7 janvier 2009.

Pédèches, P., Carnus, M.F. (2010). L'évaluation pilote du contrat didactique. Des savoirs enseignés aux savoirs évalués. Colloque international regards des didactiques disciplinaires sur les pratiques et la formation des enseignants, GRIDIFE, IUFM Midi-Pyrénées, 20-22 octobre 2010.

Terrisse, A. (2009). La didactique clinique en EPS. Origine, cadre théorique et recherches empiriques. In A. Terrisse et M.F. Carnus (dir). Didactique clinique de l'EPS, quels enjeux de savoirs ? De Boëck, Bruxelles, 13-31.

Terrisse, A., Carnus, M.F. (2009). Didactique clinique de l'EPS, quels enjeux de savoirs? De Boëck, Bruxelles.

Touboul, A., Carnus, M.F., Terrisse, A. (2010). L'influence de l'expérience et l'expertise sur l'enseignement en EPS. Colloque international regards des didactiques disciplinaires sur les pratiques et la formation des enseignants, GRIDIFE, IUFM Midi-Pyrénées, 20-22 octobre 2010. 